\title{
ON INFINITELY DIFFERENTIABLE POSITIVE DEFINITE FUNCTIONS ${ }^{1}$
}

\section{A. DEVINATZ}

1. Suppose that $f(x)$ is an infinitely differentiable positive definite function. That is to say

$$
f(x)=\int_{-\infty}^{\infty} e^{i t x} d \alpha(t),
$$

where $d \alpha(t)$ is a bounded non-negative measure. Since $f(x)$ is infinitely differentiable, it is well known (cf. C.-G. Esseen [4, p. 24]) that

$$
f^{(n)}(x)=\int_{-\infty}^{\infty} i^{n} t^{n} e^{i t x} d \alpha(t) .
$$

Therefore, the sequence $\left\{(-i)^{n} f^{(n)}(0)\right\}_{0}^{\infty}$ represents a Hamburger moment sequence. Again, if $\left\{\xi_{k}\right\}_{0}^{n}$ is an arbitrary finite set of complex numbers and $m$ is any non-negative integer, then

$$
\begin{aligned}
\left|\sum_{k=0}^{n} \xi_{k}(-i)^{k} f^{(k+m)}(x)\right|^{2} & =\left|\int_{-\infty}^{\infty} t^{m} e^{i t x} \sum_{k=0}^{n} \xi_{k} t^{k} d \alpha(t)\right|^{2} \\
& \leqq \int_{-\infty}^{\infty} t^{2 m} d \alpha(t) \int_{-\infty}^{\infty}\left|\sum_{k=0}^{n} \xi_{k} t^{k}\right|^{2} d \alpha(t) \\
& =M_{m} \sum_{r=0}^{n} \sum_{s=0}^{n} \xi_{r} \bar{\xi}_{s}(-i)^{r+s} f^{(r+s)}(0),
\end{aligned}
$$

where $M_{m}=(-i)^{2 m} f^{(2 m)}(0)$.

It turns out that if we add to these two necessary conditions a third condition, namely that $\left\{(-i)^{n} f^{(n)}(0)\right\}$ is a determined Hamburger moment sequence, ${ }^{2}$ then these three conditions are sufficient for an infinitely differentiable function to have the representation (1). However, even more is true. If $f(x)$ is defined and infinitely differentiable on some open interval containing the origin and satisfies the above conditions, then it has the representation (1); i.e. it can be extended to be a positive definite function. Since the Hamburger moment sequence is determined the extension is clearly unique (cf.

Received by the editors January 11, 1956 and, in revised form, February 15, 1956.

1 This research was supported by the United States Air Force, through the Office of Scientific Research of the Air Research and Development Command.

2 By this we mean that there exists a unique non-negative measure $d \alpha(t)$ such that $(-i)^{n} f^{(n)}(0)=\int_{-\infty}^{\infty} t^{n} d \alpha(t)$. 
Esseen [4, pp. 24-25]). Moreover, we shall show that if the Hamburger sequence is not determined, the first two necessary conditions are not sufficient. On the other hand the fact that the Hamburger sequence $\left\{(-i)^{n} f^{(n)}(0)\right\}$ is determined is in general not a necessary condition.

The theorem we shall prove in this note is as follows:

THEOREM. Let $f(x)$ be an infinitely differentiable function defined on the open interval $(-a, b)$ where $a, b>0$. If

(a) $\left\{(-i)^{k} f^{(k)}(0)\right\}_{0}^{\infty}$ is a determined Hamburger moment sequence and

(b) for every non-negative integer $m$ there exists an $M_{m}>0$ such that for every $x \in(-a, b)$ and every finite set $\left\{\xi_{k}\right\}_{0}^{n}$ of complex numbers

$$
\left|\sum_{k=0}^{n} \xi_{k}(-i)^{k} f^{(k+m)}(x)\right|^{2} \leqq M_{m} \sum_{r=0}^{n} \sum_{s=0}^{n} \xi_{r} \xi_{s}(-i)^{r+s} f^{(r+s)}(0),
$$

then there exists a bounded non-negative measure $d \alpha(t)$ such that

$$
f(x)=\int_{-\infty}^{\infty} e^{i t x} d \alpha(t)
$$

As a tool in the proof of this theorem we shall use the theory of operators in Hilbert space. This theorem was inspired by a recent result of A. P. Calderon and A. Devinatz $[2 ; 3]$ when we noticed, that after some preliminary work, the same methods as used in [2] and [3] could be used to obtain our more general result.

2. In this section we shall construct the requisite tool which we shall use in the proof of our theorem, namely a Hilbert space. To do this we consider the class $\mathcal{F}^{\prime}$ of functions of the form

$$
g(x)=\sum_{k=0}^{n} \xi_{k}(-i)^{k} f^{(k)}(x) .
$$

If $h(x)$ is another element of $\mathcal{F}^{\prime}$, namely

$$
h(x)=\sum_{k=0}^{m} \eta_{k}(-i)^{k} f^{(k)}(x),
$$

we shall construct an inner product in $\mathcal{F}^{\prime}$ by the formula

$$
(g, h)=\sum_{r=0}^{n} \sum_{s=0}^{m} \xi_{r} \bar{\eta}_{s}(-i)^{r+s} f^{(r+s)}(0) .
$$

To show that this is a well defined function, suppose that $g$ and $h$ have different representations; i.e. 
Then

$$
g(x)=\sum_{0}^{n} \xi_{k}^{\prime}(-i)^{k} f^{(k)}(x), \quad h(x)=\sum_{0}^{m^{\prime}} \eta_{k}^{\prime}(-i)^{k} f^{(k)}(x) .
$$

$$
\begin{aligned}
\sum_{r=0}^{n^{\prime}} \sum_{s=0}^{m^{\prime}} \xi_{r}^{\prime} \bar{\eta}_{s}^{\prime}(-i)^{r+s} f^{(r+s)}(0)=\sum_{r=0}^{n^{\prime}} \xi_{r}^{\prime}(i)^{r} \bar{h}^{(r)}(0) \\
=\sum_{r=0}^{n^{\prime}} \sum_{s=0}^{m} \xi_{r}^{\prime} \bar{\eta}_{s}(-i)^{r+s} f^{(r+s)}(0)=\sum_{s=0}^{m} \bar{\eta}_{s}(-i)^{s} g^{(s)}(0) \\
=\sum_{r=0}^{m} \sum_{s=0}^{m} \xi_{r} \bar{\eta}_{s}(-i)^{r+s} f^{(r+s)}(0)
\end{aligned}
$$

This shows that the bilinear function in (2) is well defined. Moreover, since $\left\{(-i)^{n} f^{(n)}(0)\right\}$ is a moment sequence, $(g, g) \geqq 0$ and by condition (b) of the theorem if $(g, g)=0$, then $g(x) \equiv 0$. Conversely if $g(x)$ $\equiv 0,(g, g)=0$. Therefore, the bilinear function defined in (2) is an actual inner product on $\mathcal{F}^{\prime}$.

In general, $\mathcal{F}^{\prime}$ is not complete with respect to this norm. We shall show that it can be completed to a Hilbert space $\mathcal{F}$ of functions on $(-a, b)$. Suppose then that $\left\{g_{n}\right\}_{1}^{\infty}$ is a Cauchy sequence in $\mathcal{F}^{\prime}$. That is to say $\left\|g_{n}-g_{m}\right\| \rightarrow 0$ as $n, m \rightarrow \infty$. By condition (b) of the theorem $\left|g_{n}(x)-g_{m}(x)\right|$ goes uniformly to zero as $n, m \rightarrow \infty$. Therefore, there exists a continuous function $g(x)$ defined on $(-a, b)$ such that $\left|g_{n}(x)-g(x)\right| \rightarrow 0$ as $n \rightarrow 0$. This extended class of functions, which we get as pointwise limits of Cauchy sequences in $\mathfrak{F}^{\prime}$, we shall designate by $\mathcal{F}$. It is clear that $\mathcal{F}$ is a linear space over the complex number field. It remains to extend the inner product from $\mathcal{F}^{\prime}$ to $\mathcal{F}$ so that $\mathcal{F}$ becomes a Hilbert space. If $g, h \in \mathcal{F}$, there exist Cauchy sequences $\left\{g_{n}\right\}$, $\left\{h_{n}\right\} \subset \mathcal{F}^{\prime}$ such that $g_{n}(x) \rightarrow g(x)$ and $h_{n}(x) \rightarrow h(x)$. We shall define

$$
(g, h)=\lim _{n \rightarrow \infty}\left(g_{n}, h_{n}\right) .
$$

That this limit exists is clear since

$$
\left|\left(g_{n}, h_{n}\right)-\left(g_{m}, h_{m}\right)\right| \leqq\left\|g_{n}-g_{m}\right\|\left\|h_{n}\right\|+\left\|g_{m}\right\|\left\|h_{n}-h_{m}\right\| .
$$

The quantities $\left\|h_{n}\right\|$ and $\left\|g_{m}\right\|$ are uniformly bounded and therefore $\left\{\left(g_{n}, h_{n}\right)\right\}$ is a Cauchy sequence. We must show yet that (3) is a well defined function.

Suppose that $\left\{\tilde{g}_{n}\right\},\left\{\tilde{h}_{n}\right\} \subset \mathcal{F}^{\prime}$ are Cauchy sequences such that $\tilde{g}_{n}(x) \rightarrow g(x)$ and $\tilde{h}_{n}(x) \rightarrow h(x)$ uniformly in $(-a, b)$. Condition (b) of the theorem tells us that for any $m, \tilde{g}_{n}^{(m)}(x) \rightarrow g^{(m)}(x)$ and $\tilde{h}_{n}^{(m)}(x)$ $\rightarrow h^{(m)}(x)$ uniformly in $(-a, b)$ and therefore, in particular, $\tilde{g}_{n}^{(m)}(0)$ 
$\rightarrow g^{(m)}(0)$ and $\tilde{h}_{n}^{(m)}(0) \rightarrow h^{(m)}(0)$. Now, clearly

$$
\lim _{n \rightarrow 0}\left(g_{n}, h_{n}\right)=\lim _{n \rightarrow \infty} \lim _{m \rightarrow \infty}\left(g_{n}, h_{m}\right)=\lim _{m \rightarrow \infty} \lim _{n \rightarrow \infty}\left(g_{n}, h_{m}\right) .
$$

Further,

$$
\begin{aligned}
\lim _{n, m \rightarrow \infty}\left(g_{n}, h_{m}\right)-\lim _{n, m \rightarrow \infty}\left(\tilde{g}_{n}, \tilde{h}_{m}\right) \\
=\lim _{m \rightarrow \infty} \lim _{n \rightarrow \infty}\left(g_{n}-\tilde{g}_{n}, h_{m}\right)+\lim _{n \rightarrow \infty} \lim _{m \rightarrow \infty}\left(\tilde{g}_{n}, h_{m}-\tilde{h}_{m}\right) .
\end{aligned}
$$

Suppose that

$$
h_{m}(x)=\sum_{k} \xi_{k, m}(-i)^{k} f^{(k)}(x)
$$

and

$$
\tilde{g}_{n}(x)=\sum_{k} \eta_{k, n}(-i)^{k} f^{(k)}(x)
$$

Then we have

$$
\lim _{m \rightarrow \infty} \lim _{n \rightarrow \infty}\left(g_{n}-\tilde{g}_{n}, h_{m}\right)=\lim _{m \rightarrow \infty} \lim _{n \rightarrow \infty} \sum_{k} \xi_{k, m}(-i)^{k}\left[g_{n}^{(k)}(0)-\tilde{g}_{n}^{(k)}(0)\right]=0
$$

and similarly

$$
\lim _{n \rightarrow \infty} \lim _{m \rightarrow \infty}\left(\tilde{g}_{n}, h_{m}-\tilde{h}_{m}\right)=\lim _{n \rightarrow \infty} \lim _{m \rightarrow \infty} \sum_{k} \eta_{k, n}(i)^{k}\left[\bar{h}_{m}^{(k)}(0)-\bar{h}_{m}^{(k)}(0)\right]=0 .
$$

This shows that the function defined in (3) is indeed well defined.

To show that this bilinear function is an inner product we first note that for every $g$ in $\mathcal{F}$ there exists a Cauchy sequence $\left\{g_{n}\right\} \subset \mathcal{F}^{\prime}$ such that

$$
(g, g)=\lim _{n \rightarrow \infty}\left(g_{n}, g_{n}\right) \geqq 0 .
$$

Again, suppose that for $g \in \mathcal{F},(g, g)=0$. Suppose $\left\{g_{n}\right\} \subset F^{\prime}$ is a Cauchy sequence such that $g_{n}(x) \rightarrow g(x)$ and $(g, g)=\lim \left(g_{n}, g_{n}\right)=0$. Then since

$$
\left|g_{n}(x)\right|^{2}=\left|\sum_{k} \xi_{k, n}(-i)^{k} f^{(k)}(x)\right|^{2} \leqq M_{0}\left\|g_{n}\right\|^{2}
$$

we have that $g_{n}(x) \rightarrow 0$, which shows that $g(x) \equiv 0$. On the other hand if $g(x) \equiv 0$ on $(-a, b)$ then $g \in \mathcal{F}^{\prime}$ and $(g, g)=0$. Therefore, $\mathcal{F}$ forms a linear space with an inner product. The proof of the fact that $\mathcal{F}$ is complete uses standard arguments and we leave this to the reader.

An important fact that we shall need in the future, a fact used 
previously in connection with the space $\mathcal{F}^{\prime}$, is that for any $g \in \mathcal{F}$

$$
(-i)^{n} g^{(n)}(0)=\left(g(x),(-i)^{n} f^{(n)}(x)\right) .
$$

This can be easily proved by taking a Cauchy sequence $\left\{g_{n}(x)\right\} \subset F^{\prime}$ such that $g_{n}(x) \rightarrow g(x)$, noting that (4) is true for every $g_{n}$ in this sequence and then passing to the limit.

3. Now that we have constructed the Hilbert space $\mathcal{F}$ we can proceed with the proof of our theorem. First we wish to set up a conjugation operator on $\mathcal{F}$. Consider first an element $g \in \mathcal{F}^{\prime}$; i.e. $g(x)$ $=\sum_{0}^{n} \xi_{k}(-i)^{k} f^{(k)}(x)$. Define

$$
J g(x)=\sum_{0}^{n} \bar{\xi}_{k}(-i)^{k} f^{(k)}(x) .
$$

Now, $J^{2} g(x)=g(x)$ and if $h(x)=\sum_{0}^{m} \eta_{k}(-i)^{k} f^{(k)}(x)$, then

$$
(J g, J h)=\sum_{r=0}^{n} \sum_{s=0}^{m} \bar{\xi}_{r} \eta_{s}(-i)^{r+s} f^{(r+s)}(0)=(h, g) .
$$

Since $\mathcal{F}^{\prime}$ is dense in $\mathcal{F}, J$ can be extended to all of $\mathcal{F}$ and is a conjugation operator.

Define an operator $D$, with domain $\mathcal{F}^{\prime}$, by the relation

$$
D g(x)=-i d g(x) / d x .
$$

In other words, if $g(x)=\sum_{0}^{n} \xi_{k}(-i)^{k} f^{(k)}(x)$, then

$$
D g(x)=\sum_{0}^{n} \xi_{k}(-i)^{k+1} f^{(k+1)}(x) .
$$

If $h(x)=\sum_{0}^{m} \eta_{k}(-i)^{k} f^{(k)}(x)$, then

$$
(D g, h)=\sum_{r=0}^{n} \sum_{s=0}^{m} \xi_{r} \bar{\eta}_{s}(-i)^{r+s+1} f^{(r+s+1)}(0)=(g, D h) .
$$

Therefore, $D$ is a symmetric operator. Further, since it clearly permutes with $J$, it has a self-adjoint extension. We shall show that the self-adjoint extension is unique and is therefore the closure of $D$.

Suppose $H$ is any self-adjoint extension of $D$, and $d E(t)$ its canonical resolution of the identity. If $f_{0}(x)=f(x)$, then

$$
(-i)^{n} f^{(n)}(0)=\left(H^{n} f_{0}, f_{0}\right)=\int_{-\infty}^{\infty} t^{n} d\left(E(t) f_{0}, f_{0}\right) .
$$

Since by hypothesis $\left\{(-i)^{n} f^{(n)}(0)\right\}$ is a uniquely determined Hamburger moment sequence, the measure $d\left(E(t) f_{0}, f_{0}\right)$ is uniquely deter- 
mined. Suppose we let $f_{n}(x)=(-i)^{n} f^{(n)}(x)$. The linear manifold generated by this class of elements is dense in $\mathcal{F}$. Now,

$$
\left(E(\lambda) f_{n}, f_{m}\right)=\left(E(\lambda) H^{n+m} f_{0}, f_{0}\right)=\int_{-\infty}^{\lambda} t^{n+m} d\left(E(t) f_{0}, f_{0}\right) .
$$

Therefore, for any $n$ and $m,\left(E(\lambda) f_{n}, f_{m}\right)$ is uniquely determined in the sense that if $H_{1}$ is another self-adjoint extension of $D, d E_{1}(t)$ its canonical spectral measure, then $\left(E_{1}(\lambda) f_{n}, f_{m}\right)=\left(E(\lambda) f_{n}, f_{m}\right)$. This means however that $D$ has only one self-adjoint extension, namely its closure.

What we have just proved means that $D^{*}$, the adjoint of $D$, is selfadjoint and is the closure of $D$. Therefore $g$ is in the domain of $D^{*}$ if and only if there exists a sequence $\left\{g_{n}\right\} \subset \mathcal{F}^{\prime}$ such that $g_{n} \rightarrow g$ and $D g_{n} \rightarrow D^{*} g$ in the strong topology of $\mathcal{F}$. This implies uniform pointwise convergence and therefore,

$$
D^{*} g(x)=-i d g(x) / d x .
$$

Let us consider the group of unitary operators

$$
U_{x}=\int_{-\infty}^{\infty} e^{i t x} d E(t),
$$

where $d E(t)$ is the canonical spectral measure of $D^{*}$. Let $g \in \mathcal{F}$ be such that

$$
U_{x} g=\int_{-c}^{c} e^{i t x} d E(t) g,
$$

where $c$ is a positive finite number. It is clear that any such element belongs to the domain of $D^{*}$. Let us expand $e^{i t x}$, as a function of $t$, in its Taylor series about the origin. Since this Taylor series is uniformly convergent in any finite interval we have for every $x$

$$
\begin{aligned}
U_{x} g & =\sum_{0}^{\infty} \frac{x^{n}}{n !} \int_{-c}^{c} i^{n} t^{n} d E(t) g \\
& =\sum_{0}^{\infty} \frac{x^{n}}{n !} i^{n} D^{* n} g
\end{aligned}
$$

where the convergence is in the strong topology of $\mathcal{F}$. But since convergence in the strong topology implies pointwise convergence we have for every $y \in(-a, b)$

$$
U_{x} g(y)=\sum_{0}^{\infty} \frac{g^{(n)}(y)}{n !} x^{n} .
$$


Since this series has an infinite radius of convergence for every $y \in(-a, b)$ it is well known (cf. R. P. Boas [1]) that $g(y)$ is analytic. Therefore if $x+y \in(-a, b)$

$$
U_{x} g(y)=g(y+x) .
$$

Since the class of elements for which (5) holds is dense in $\mathcal{F},(5)$ must hold for every element of $\mathcal{F}$. If again we set $f_{0}(x)=f(x)$ then by means of the relationship (4) we get

$$
f(x)=U_{x} f(0)=\left(U_{x} f_{0}, f_{0}\right)=\int_{-\infty}^{\infty} e^{i t x} d\left(E(t) f_{0}, f_{0}\right) .
$$

This completes the proof of the theorem.

4. In this section we shall show that there exist functions $f(x)$ such that $\left\{(-i)^{n} f^{(n)}(0)\right\}$ is an undetermined Hamburger moment sequence and which satisfy condition (b) of our theorem but which are not positive definite. This is essentially the same example as given in [3].

Let $\left\{\mu_{n}\right\}_{0}^{\infty}$ be any undetermined Hamburger moment sequence. Then there exist two different bounded non-negative measures $d \alpha_{1}(t)$ and $d \alpha_{2}(t)$ such that

$$
\mu_{n}=\int_{-\infty}^{\infty} t^{n} d \alpha_{1}(t)=\int_{-\infty}^{\infty} t^{n} d \alpha_{2}(t)
$$

Let

$$
\begin{aligned}
& f_{1}(x)=\int_{-\infty}^{\infty} e^{i t x} d \alpha_{1}(t), \\
& f_{2}(x)=\int_{-\infty}^{\infty} e^{i t x} d \alpha_{2}(t) .
\end{aligned}
$$

Further, let

$$
f(x)=\left\{\begin{array}{lll}
f_{1}(x) & \text { for } x \geqq 0 \\
f_{2}(x) & \text { for } \quad x \leqq 0
\end{array}\right.
$$

Then, $(-i)^{n} f^{(n)}(0)=\mu_{n}$ and condition (b) of the theorem is clearly satisfied. However, $f(x)$ cannot be a positive definite function since a positive definite function must satisfy the relation

$$
f(x)=\bar{f}(-x) .
$$

However, we would get in this case

$$
f_{1}(x)=\bar{f}_{1}(-x)=\bar{f}_{2}(-x)=f_{2}(x)
$$

which would mean $d \alpha_{1}=d \alpha_{2}$. 


\section{REFERENCES}

1. R. P. Boas, $A$ theorem on analytic functions of a real variable, Bull. Amer. Math. Soc. vol. 41 (1935) pp. 233-236.

2. Alberto Calderon and Allen Devinatz, Sur certaines courbes dans l'espace de Hilbert, C. R. Acad. Sci. Paris vol. 241 (1955) pp. 539-541.

3. - Sur certaines courbes d courbure constante dans l'espace de Hilbert, C. R. Acad. Sci. Paris vol. 241 (1955) pp. 586-587.

4. Carl-Gustave Esseen, Fourier analysis of distribution functions, Acta Math. vol. 77 (1945) pp. 1-125.

UNIVERSITY OF CONNECTICUT AND

WASHINGTON UNIVERSITY

\section{ON A SERIES OF RAINVILLE INVOLVING LEGENDRE POLYNOMIALS}

B. R. BHONSLE

1. The object of this paper is to obtain some relations involving Legendre polynomials with the help of a series given by E. D. Rainville. The results are believed to be new.

2. We start with the series given by E. D. Rainville

$$
P_{n}(\cos \alpha)=\left(\frac{\sin \alpha}{\sin \beta}\right)^{n} \sum_{k=0}^{n} c_{n, k}\left[\frac{\sin (\beta-\alpha)}{\sin \alpha}\right]^{n-k} P_{k}(\cos \beta) .
$$

Putting $\beta=2 \alpha$ and $\cos 2 \alpha=x$, we get

$$
2^{n / 2}(1+x)^{n / 2} P_{n}\left(\left(\frac{1+x}{2}\right)^{1 / 2}\right)=\sum_{k=0}^{n} c_{n, k} P_{k}(x) .
$$

From (2.2) and the orthogonal property

$$
\begin{aligned}
\int_{-1}^{1}(1+x)^{n / 2} P_{r}(x) P_{n}\left(\left(\frac{1+x}{2}\right)^{1 / 2}\right) d x & \\
=\frac{c_{n, \gamma}}{2^{n / 2-1}(2 \gamma+1)}, & 0 \leqq \gamma \leqq n, \\
=0, & r>n .
\end{aligned}
$$

Using (2.3) with Adams' expansion (Modern analysis, p. 331) for

Received by the editors October 2, 1955 and, in revised form, March 26, 1956. 